\title{
New mechanism of dust growth and gravitation-like instabilities in astrophysical plasmas
}

\author{
R. Bingham ${ }^{1,2}$ and V. N. Tsytovich ${ }^{3,4}$ \\ 1 Rutherford Appleton Laboratory, Chilton, Didcot, Oxon OX11 0QX, UK \\ 2 University of Strathclyde, Glasgow G1 1XQ, Scotland, UK \\ 3 General Physics Institute, Russian Academy of Science, Vavilova Str. 38, Moscow, Russia \\ 4 Moscow Physical Technical Institute, Moscow, Russia
}

Received 19 January 2001 / Accepted 3 August 2001

\begin{abstract}
Dust growth by agglomeration due to a new mechanism of dust attraction is shown to compete in space plasmas with the standard accretion mechanism by neutral particles. We demonstrate that dust growth by accretion of neutrals and plasma particles is aided by a pressure force due to a shadowing effect first proposed (Bingham \& Tsytovich 2001) to explain rapid growth of dust in laboratory discharges. Consequences of this new force promoting dust growth by agglomeration in astrophysics are discussed including the role of a new gravitational-like instability which produces clumping enhancing the rate of gravitational collapse.
\end{abstract}

Key words. dust - agglomeration: plasma: gravitational collapse

\section{Introduction}

The subject of dust formation in space and astrophysical plasmas is important in diverse environments such as dust molecular clouds, proto-planetary nebulae, stellar outbursts, and supernova explosions. The formation of dust proceeds the formation of stellar objects and planets. And the ultimate fate of most of the dust is to be consumed in the process of making stars, planets and smaller bodies such as comets and asteroids.

In all these environments the dust particles interact with both neutral and plasma particles as well as with (ultraviolet) radiation and cosmic rays. The conventional view of grain growth is one based on accretion where condensation nuclei containing a small number of atoms formed in the process of adiabatic expansion bound together by the Van der Waals and chemical forces grow by atoms sticking and attaching themselves to the surface (Watson \& Salpeter 1972; Spitzer 1978). This first stage of Brownian motion-driven dust growth was considered in detail both theoretically and numerically (Weidenschilling \& Cuzzi 1993; Kempf et al. 1999; Meaking 1991) and confirmed recently by micro-gravity experiments (Blum et al. 2000). This model has also been used to explain the slow growth of dust grains to a size of about $0.1 \mu \mathrm{m}$ in $10^{6}-10^{9}$ years. The problem in producing larger dust grains by accretion is due to a number of effects including, the sticking probability which reduces with increasing size, the ambient density and temperature of neutrals

Send offprint requests to: R. Bingham,

e-mail: R.Bingham@rl.ac.uk and plasma as well as the temperature of the dust grain itself. High grain temperatures prevent further growth. Observations (Winters et al. 2000) of dust reveal typical sizes of the order of $10 \mu \mathrm{m}-1000 \mu \mathrm{m}$. And even more importantly recent observations (Kempf et al. 1999) of circumstellar dust shells around long-period variables (Winters et al. 2000) demonstrate dust formation taking place on a time scale of 10 years. The winds of these C-stars are so effective at producing dust that the circumstellar dust shell totally obscures the central object. Multiple dust shells are also observed forming the circumstellar structure. Given the rate of production and multiple shell like structures we propose a new process of dust formation in astrophysical plasmas namely agglomeration. An important feature of the light curve from supernova SN 1987A is a break at about 500 to 600 days coincident with an increase in the infrared flux and an increase in absorption of red-shifted emission lines (Chevalier 1992). These observations confirm the formation of dust within the supernova eject after about 500 days. The dust in interstellar space can be studied using telescopes. Small particles of solid matter tend to polarize light reflected and scattered by their surfaces. Particles of about a micron in size appear brighter in forward-scattered light than in back-scattered light.

Dust agglomeration has been shown to be important in laboratory etching experiments (Garscadden et al. 1994) where growth of dust is extremely rapid and is due in the final stages to dust-dust attraction by a plasma or neutral bombardment force known as the shadow force (Tsytovich et al. 1996a,b). Since small grains of dust are 
attaching themselves together the rate of growth to large sized grains of order $10 \mu \mathrm{m}$ or larger is extremely rapid of order of hours in laboratory experiments and as we will demonstrate it is of order 10 years in C-stars.

This rapid formation of large dust grains through agglomeration can lead to a new type of instability formed as a result of the net attractive force existing between the dust grains. By replacing the force of gravity by this force we derive a linear dispersion relation describing the growth rate of a gravitation like instability leading to clumping of the dust structures with a typical length for collapse smaller than the Jeans length for gravitational collapse. We find that the formation of clump like structures proceeds faster than gravitational collapse. The consequence is that this type of collapse is perhaps necessary before the onset of gravitational collapse resulting in a self similar or scale invariant process describing self-organization of dust structures.

The shadow force that is responsible for this phase of collapse is due to an anisotropic pressure of neutrals and plasma particles on the surface of a dust grain the shadow or shielding by a neighbouring grain. The shielding effect results in a reduction in pressure between the grains forcing them to coalesce or agglomerate. The force produced as a result of the pressure differential is proportional to the square of the ratio of dust size to separation distance. At large separation distances $r$ this force is larger than the Van der Waals force which is proportional to $r^{-8}$.

In this article we estimate the rate of dust growth due to agglomeration and compare it with the normal accretion model. We will also demonstrate that it can lead to a rapid gravitational like collapse with a length much smaller than the Jeans length for gravitational collapse. The result is the formation of dust clumps.

\section{Dust growth}

Our understanding of dust growth has benefited greatly from laboratory experiments (Garscadden et al. 1994; Boufendi \& Bouchoule 1994) carried out within the last 10 years. These experiments have been successfully explained by a combination of a series of stages of dust growth. In the first stage nucleation occurs with the chemical formation of atom clusters this stage is well documented for both laboratory and space scenarios. It is understood that the Van der Waals and chemical binding forces play a key role in forming these nm sized molecular clusters. One advantage in space is that gases from outbursts or winds from stars, supernova explosions or jets are adiabatically cooled to very low temperatures forming atom clusters when the attaching (sticking) coefficient is of the order of the de-attaching (evaporation) coefficient for neutral gas atoms. Gas jet experiments also confirm the formation of atom clusters. In previous calculations of dust growth in space these atom clusters accrete single neutral atoms through collisions. Neutral gas accretion requires dust temperatures of order $10-80 \mathrm{~K}$, the dust can easily maintain these temperatures by radiation cooling.
In molecular clouds dust at this temperature has been detected, however, for the dust to grow to the size of several microns (Spitzer 1978) takes about $10^{6}-10^{9}$ years by the process of accretion. This may be satisfactory to explain the observations of dust in molecular clouds but in outbursts, stellar winds and supernova explosions the dust formation is extremely rapid. The main difference between these regions of dust formation and molecular clouds is the presence of plasma which is known to assist in the process of dust growth. Laboratory etching experiments are carried out in plasmas where the temperature can be as high as $3 \times 10^{4} \mathrm{~K}$ and the formation of atom clusters up to $10 \mathrm{~nm}$ are still observed, these conditions are closer to those of outbursts, stellar winds and supernova explosions than to molecular clouds. The main difference being the absence of $3 \times 10^{4} \mathrm{~K}$ plasma in the molecular clouds (the degree of ionization by cosmic rays or radioactivity is less than $10^{-7}$ ). The exception is at boundaries illuminated by UV radiation resulting in rapid growth in several stages, where the presence of a plasma changes the growth processes considerably.

The second stage observed in laboratory experiments carried out in the presence of a plasma is rapid dust agglomeration up to grain sizes of order $0.1-1.0 \mu \mathrm{m}$. One of the main differences introduced by the plasma is the presence of charge on the grains which normally acquire a negative charge in the absence of UV radiation due to the greater electron mobility. Estimates show that for grains of $10 \mathrm{~nm}$ in size charges of 10-50 electron charges are possible on isolated grains. At the agglomeration stage the dust density is high which has the effect of decreasing the charge on the grains to about $3-20$ electrons. The shadow force produced by the pressure anisotropy can easily overcome the Coulomb repulsion between the grains leading to agglomeration of more and more grains forming larger and lower dust densities. The growth through agglomeration has two effects; to reduce the dust density and increase the charge on the dust to about $10^{3}$ electrons which is large enough to overcome the shadow force through Coulomb repulsion slowing the growth (Boufendi \& Bouchoule 1994).

The third stage of growth is the plasma deposition or plasma accretion stage where ions are attracted to the charged dust, increasing the size of dust to about $100 \mu \mathrm{m}$ or larger. During this stage irregular shaped dust soon becomes spherical due to a spherical converging flow of ions. The main difference between neutral accretion and ion accretion or deposition is that the ions are accelerated by the field produced by the charged dust to energies of the order of the electron temperature. The potential of the charged dust grain is of the order of the electron thermal energy in some cases this can be as high as $3 \times 10^{4} \mathrm{~K}$. This energy which is imparted to the ions causes ion implantation. The ion can be embedded deep within the dust in contrast to neutral particles which only sit on the surface. The presence of the plasma inevitably produces a continuous flux of ions which enhances the growth phase. For low levels of ionization the flux of neutrals can be much larger 
than the flux of ions. Contrary to ions the neutrals have a very short residence time on the dust unless the temperature of the dust is extremely low of order $10 \mathrm{~K}$ (Spitzer 1978). At higher temperatures greater than $100 \mathrm{~K}$ the accretion of neutrals will not occur but accretion by ions will be rapid. In laboratory plasmas where the ion density is of order $10^{9} \mathrm{~cm}^{-3}$ and electron ion temperature ratio $T_{\mathrm{e}} / T_{\mathrm{i}} \sim 10^{2}$, the ion deposition time for $50 \mu \mathrm{m}$ sized particles is about $30 \mathrm{~min}$ i.e. relatively fast, indicating that the ion implantation (bombardment) mechanism should always be considered for these conditions and needs to be considered for astrophysical conditions.

The shadow force is the main force which acts in the fourth stage of dust growth. The shadow force due to neutrals and ions caused by dust is given by a simple expression which can easily be obtained qualitatively (for a more exact theory see Bingham \& Tsytovich 2001; Khodataev et al. 2001).

$$
F=\frac{a^{2}}{r^{2}} n T \pi a^{2} A
$$

where $a^{2} / r^{2}$ is the solid angle, $a$ is the dust size and $r$ is the distance separating the dust particles, $n T$ is the pressure of either neutrals or ions we can neglect electrons because of their smaller mass and $A$ is a numerical coefficient of order of unity. In the calculation we have assumed that the dust particles are of equal size.

The physics of the shadow force is very simple. For a single dust particle the flux of absorbed charged and neutral particles on the dust surface is symmetric resulting in no net momentum transfer. Another dust particle at distance $r$ shadows the flux to the first dust particle with a solid angle $\approx a^{2} / r^{2}$. The net momentum transfer will be proportional to the solid angle, to the surface area of the dust particle $\pi a^{2}$ and to the pressure $n T$ as written in Eq. (1). The numerical coefficient $A$ depends on whether the distance between the dust particle is less or larger than the mean free path of dust/neutral or dust/ion collisons being in both cases of the order of 1 (Khodataev et al. 2001). In the case where the distance between dust particles is larger than the mean free path the physics of the force is slightly different, one dust particle creates a temperature gradient in the neutral gas component and another dust particle is attracted by the thermophoretic force due to this temperature gradient (Khodataev et al. 2001). The attraction exists if the dust surface temperature is less than the temperature of the neutral gas, which is almost always the case in optically thin dust clouds where the dust surface is cooled by radiation losses. The energy flux of ions on dust particles which heats the dust surface is usually less than the radiation losses by more than 15 orders of magnitude and the heating by other types of radiation is less than the cooling for optically thin clouds. The shadow force is inversely proportional to $r^{2}$ as for gravity and Coulomb forces, it depends on the neutral $n_{\mathrm{n}}$ or ion density $n_{\mathrm{i}}$ and their temperature $T$.

The fourth stage of dust growth observed under laboratory conditions but not all situations
(Garscadden et al. 1994) is the advanced stage or second stage of agglomeration which leads to dust grains of $0.1 \mathrm{~mm}$ in size which are observed optically. The physics of this stage is dominated by dust-dust attraction, which is proportional to the fourth power of the dust size, over-coming Coulomb repulsion between the charged dust, which is proportional to the square of the dust size (Tsytovich et al. 1996a,b).

The four stages of dust growth observed in laboratory experiments in the presence of plasma will also be important in space plasmas. For example in carbon rich star outbursts (Winters et al. 2000) dust formation takes about 10 years in regions where there is a mixture of neutrals and plasma at relatively high temperatures. The gas density in these stellar outbursts is as high as $10^{10} \mathrm{~cm}^{-3}$ with temperatures large enough that ionization is also present. The dust shells in these carbon rich stars form within 5 stellar radii in regions where the temperature is about $1000 \mathrm{~K}$ and it is expected that the four stages of dust formation should proceed including ion implantation and agglomeration. In a gas or plasma the forces transmitted to the dust grains are the random forces transferred to the small particles during Brownian motion. If we bias the collisions so that they are not isotropic for example through shielding, results in the shadow force given by Eq. (1).

In previous studies of dust growth in space, Eq. (1) is not considered only the accretion of neutrals is assumed and isotropic collisions with dust grains results in no net force between the grains. This is also true if the neutral atom attaches itself and then de-attaches with the same temperature. If the neutral atom during its contact with the surface has sufficient time to thermalize to the dust temperature and is emitted with the energy corresponding to the dust temperature then the factor $\left(1-\sqrt{\frac{T_{\mathrm{ds}}}{T_{\mathrm{n}}}}\right)$ (Khodataev et al. 2001) appears in the expression for the force given by Eq. (1), where $T_{\mathrm{ds}}$ is the dust surface temperature and $T_{\mathrm{n}}$ is the neutral temperature. The force vanishes for $T_{\mathrm{ds}}=T_{\mathrm{n}}$ and is largest for $T_{\mathrm{ds}} \ll T_{\mathrm{n}}$ a condition often found in space where the dust cools by emission of radiation at the rate $\pi a^{2} \sigma_{\mathrm{SB}} T_{\mathrm{ds}}^{4}$, where $\sigma_{\mathrm{SB}}$ is StefanBoltzmann constant. In this case the dust cloud is optically thin for radiation from dust a situation found in dust-molecular clouds. In Khodataev et al. (2001) it was shown that in an optically thin dust cloud the dust temperature is always smaller than the gas temperature, the forces between dust particles due to gas particle bombardment are always attractive and that with an increase of the gas density the attraction force "saturate" at densities of about $10^{16} \mathrm{~cm}^{-3}$ much larger than in any dust-molecular cloud.

The force given by Eq. (1) operates in the presence or absence of plasmas. In the presence of plasma the force due to the ion and neutral flux should be compared to the Coulomb force $F_{\mathrm{c}}$ between two dust grains given by

$F_{\mathrm{c}}=\frac{Z_{\mathrm{d}}^{2} e^{2}}{r^{2}}=\left(\frac{Z_{\mathrm{d}} e^{2}}{a T_{\mathrm{e}}}\right)^{2} \frac{n_{\mathrm{i}} T_{\mathrm{e}}^{2} a^{2}}{e^{2} n_{\mathrm{i}} r^{2}}=z^{2} \frac{a^{2}}{r^{2}} n_{\mathrm{i}} T_{\mathrm{e}} 4 \pi d_{\mathrm{e}}^{2}$ 
where $z=\frac{Z_{\mathrm{d}} e^{2}}{a T_{\mathrm{e}}}$ is the dimensionless dust charge of order $1-2$ and $d_{\mathrm{e}}=\sqrt{\frac{T_{\mathrm{e}}}{4 \pi n_{\mathrm{e}} e^{2}}}$ is the electron Debye radius. Comparison of Eqs. (1)-(2) demonstrates that the bombardment force due to ions is $\frac{4 d_{\mathrm{e}}^{2} z^{2}}{a^{2}}$ less than the Coulomb repulsive force for $r \ll d_{\mathrm{e}}$. For $r \gg d_{\mathrm{e}}$ the Coulomb force is screened while the attractive shadow force is not, this can lead to a contraction of the dust cloud until the interdust distances are comparable to 10 times the Debye radius. For distances larger than the Debye radius the ion accretion force (Tsytovich et al. 1996a,b) can dominate Coulomb repulsion and should be added to the attraction due to gas particle bombardment but this contribution is usually small. For outbursts of carbon rich stars where $n_{\mathrm{n}} \simeq 10^{10} \mathrm{~cm}^{-3}, n_{\mathrm{i}}$ is of order $n_{\mathrm{n}}$ and $T_{\mathrm{e}} \sim 1 \mathrm{eV}$, resulting in a Debye radius of between 100 and $1000 \mu \mathrm{m}$, attraction can occur between two equally charged grains. Using $r \sim 4 d_{\mathrm{e}}$ where the repulsive force can be overcome by the attractive force forming an attractive potential well, with the potential given by

$V \simeq F_{\mathrm{c}} r \approx z^{2} n_{\mathrm{i}} d_{\mathrm{e}} a^{2} T_{\mathrm{e}}$

We find that a "dust molecule" is formed if

$T_{\mathrm{d}}<T_{\mathrm{e}} n_{\mathrm{i}} d_{\mathrm{e}} a^{2} z^{2}$

where $T_{\mathrm{d}}$ is the dust kinetic temperature not the surface temperature. In environments where dust is cooled by emission of radiation condition (4) can easily be satisfied. For $n_{\mathrm{i}} \sim 10^{10} \mathrm{~cm}^{-3}, d_{\mathrm{e}} \sim 10^{-2} \mathrm{~cm}$ i.e. $r \sim 10^{3} \mu \mathrm{m}$ and $a \sim 1 \mu \mathrm{m}$ Eq. (4) results in $T_{\mathrm{d}}<T_{\mathrm{e}} z^{2} \sim 10 T_{\mathrm{e}}$ which is easily satisfied in carbon rich star outbursts (Winters et al. 2000).

Laboratory experiments (Thomas \& Morfill 1996) demonstrate the possibility of producing regular dust structures known as dust crystals which occur for $Z_{\mathrm{d}} e^{2} n_{\mathrm{d}}^{\frac{1}{3}}>170 T_{\mathrm{d}}$. In space this condition is more difficult to satisfy namely

$T_{\mathrm{d}}>T_{\mathrm{e}} n_{\mathrm{i}} a d_{\mathrm{e}}^{2} z^{2}\left(n_{\mathrm{d}}^{\frac{1}{3}} a\right) / 170$

but it may be possible which has lead to suggestions that dust crystals could exist in space environments (Morfill 2000). For $T_{\mathrm{i}} \ll T_{\mathrm{e}}$ numerical results (Bingham $\&$ Tsytovich 2001) show that the attraction force Eq. (1) can dominate the Coulomb force for $a>(8-60) d_{\mathrm{i}}$ thus leading to dust agglomeration, $d_{\mathrm{i}}$ is the ion Debye radius.

In dust molecular clouds $d_{\mathrm{e}}$ is very large and the degree of ionization is very low, however, cosmic rays produce ionization such that $\frac{n_{\mathrm{i}}}{n_{\mathrm{d}}} \sim 10^{-6}-10^{-8}$ which is only about one order of magnitude less than in laboratory experiments. In most laboratory experiments the neutral and ion shadow forces are of the same order of magnitude and give rise to the second and the fourth stages of agglomeration. In dust-molecular clouds if the dust is not radioactive the main attraction force is determined by the neutral flux and Eq. (1) can be used if the dust surface temperature is lower than the gas temperature. The phenomenon of dust agglomeration due to neutral particle flux bombardment was not previously considered for dust-molecular clouds. It can operate effectively for a range of temperatures above the critical temperature range of $10-80 \mathrm{~K}$ for gas accretion. At larger temperatures this will serve as the main mechanism responsible for dust growth. An estimate of the agglomeration time is found by taking into account the relative number of particles in phase space with energies less than the attractive potential well

$$
\frac{\mathrm{d} m_{\mathrm{d}}}{\mathrm{d} t}=2 m_{\mathrm{d}} v_{\mathrm{d}} n_{\mathrm{d}}^{\frac{1}{3}}\left(\frac{V}{T_{\mathrm{d}}}\right)^{\frac{3}{2}} ; \quad v_{\mathrm{d}}=\sqrt{\frac{T_{\mathrm{d}}}{m_{\mathrm{d}}}} .
$$

Since the force acting is in the direction separating the two dust particles we can use for the time scale the time for the dust to travel the inter-dust distance which is a direct consequence of (6) given by

$$
\frac{1}{\tau_{\text {aggl. }}} \simeq \sqrt{\frac{T_{\mathrm{d}}}{m_{\mathrm{d}}}} n_{\mathrm{d}}^{\frac{1}{3}}\left(\frac{T_{\mathrm{e}} n_{\mathrm{i}} a^{2} d_{\mathrm{e}} z^{2}}{T_{\mathrm{d}}}\right)^{3 / 2}
$$

for $a \sim 1 \mu \mathrm{m}, T_{\mathrm{d}} \sim 100 \mathrm{~K}, n_{\mathrm{d}} \sim 10^{-3} \mathrm{~cm}^{-3}$ we estimate $\tau_{\text {aggl. }}$ to be about $10^{9} \mathrm{~s} \simeq 30$ years.

\section{Gravitational like instability}

The shadow force also creates a gravitational like instability. The dispersion relation for the agglomeration instability for the dust component can easily be written using the dispersion relation for the Jeans instability by replacing the force of gravity by the shadow force resulting in

$\omega^{2}=k^{2} v_{\mathrm{s}}^{2}-G_{\mathrm{eff}} n_{\mathrm{d}} m_{\mathrm{d}}$

where according to (1)

$G_{\mathrm{eff}}=\frac{n T_{\mathrm{n}} \pi a^{4}}{m_{\mathrm{d}}^{2}}$

and

$v_{\mathrm{s}}^{2}=\frac{n_{\mathrm{d}} T_{\mathrm{d}}+n_{\mathrm{n}} T_{\mathrm{n}}}{n_{\mathrm{d}} m_{\mathrm{d}}+n_{\mathrm{n}} m_{\mathrm{n}}} \sim \frac{n_{\mathrm{n}} T_{\mathrm{n}}}{n_{\mathrm{d}} m_{\mathrm{d}}+n_{\mathrm{n}} m_{\mathrm{n}}}$

if $n_{\mathrm{n}} T_{\mathrm{n}} \gg n_{\mathrm{d}} T_{\mathrm{d}}$. Although the attraction forces are acting only on dust particles the expression for $v_{\mathrm{s}}$ will correspond to the sound speed for frequencies much less than the dustneutral collision frequency (Tsytovich 1997).

The corresponding Jeans type length is determined by the relation

$L=\frac{2 \pi v_{\mathrm{s}}}{\sqrt{G_{\mathrm{eff}} n_{\mathrm{d}} m_{\mathrm{d}}}} \simeq \frac{2 \sqrt{\pi}}{n_{\mathrm{d}} a^{2} \sqrt{\left(1+n_{\mathrm{n}} m_{\mathrm{n}} / n_{\mathrm{d}} m_{\mathrm{d}}\right)}}$.

For $a \sim 1 \mu \mathrm{m}, n_{\mathrm{d}} \sim 10^{-3} \mathrm{~cm}^{-3}$ we have $L \sim 10^{11} \mathrm{~cm}$, resulting in dust clumps of order $10^{11}-10^{12} \mathrm{~cm}$ in size.

These clumps are much smaller than the Jeans length for gravitational collapse and will proceed stellar and planetary formation. It is envisaged that clumping could proceed through this mechanism forming larger and larger scales all the way to the Jeans length scales, at each scale 
the structures are similar and scale invariant. These structures are a result of self-organization found in complex physical systems, each scale could collapse forming structures from asteroids in size all the way up to star formation which could be enhanced by additional compression produced by shock waves. The characteristic time for the Jeans like instability to develop is given by $\tau_{\text {ag.inst }}$ i.e. the agglomeration instability time scale

$\tau_{\text {ag.inst }} \simeq \sqrt{\frac{m_{\mathrm{d}}}{n_{\mathrm{n}} T_{\mathrm{n}} \pi a^{4} n_{\mathrm{d}}}}$

which for $n_{\mathrm{d}} \sim 10^{-3} \mathrm{~cm}^{-3}, n_{\mathrm{n}} \sim 10^{3}, T_{\mathrm{n}} \sim 50 \mathrm{~K}, a \sim$ $1-3 \mu \mathrm{m}$ is

$\tau_{\text {ag.inst }} \sim 10-100$ years.

This is 3 orders of magnitude faster than the Jeans gravitational collapse time and the Jeans length is $10^{3}$ orders of magnitude larger. The result is a clumpy structure which is often observed in dust-molecular clouds.

\section{Conclusions}

Recent experiments (Blum et al. 2000) demonstrated dust agglomeration under micro-gravity conditions. It should be emphasized that the terminology for dust growth varies from paper to paper. In this paper we discuss growth of large dust grains in a high density regime whereas in (Blum et al. 2000) the growth is in a lower density regime where the term agglomeration is used for the case where Van der Waals force at small distances overcomes the repulsion causing the sticking of dust particles. At higher densities our effect dominates through Eq. (1) which is larger than the Van der Waals force which varies as $1 / r^{8}$. The agglomeration discussed in Blum et al. (2000) is in our terminology the first chemical stage of dust clumping forming clumps containing many molecules (second stage).

We emphasized here the possibility of the existence of a fourth stage similar to that in laboratory experiments, even in a completely neutral gas the shadow force operates producing dust particles of the size $10 \mu \mathrm{m}$ or larger. In many cases the presence of ionization can also be important for dust growth. There is the possibility to check our mechanism in micro-gravity experiments. The experiments already performed deal with very low neutral gas pressure where according to our estimate the potential $V$ given by Eq. (3) is less than that due to the Van der Waals force. To check our results it is desirable to work with larger sized dust particles and with a larger neutral gas pressure. In present experiments the attraction force Eq. (1) can help dust clumping at large distances, where the force given by Eq. (1) over-comes the Van der Waals force.

In this article we have described a new force of attraction giving rise to rapid dust agglomeration in astrophysical plasmas, the rate of dust formation is much more rapid than that due to the accretion of neutral particles. This new force arises due to a shadowing of the dust particles in either a neutral or plasma atmosphere. The force of attraction that results from the shadow effect gives rise to the formation of smaller scale structurs on faster time scales than that due to the standard Jeans instability. The structures that are then formed could easily be responsible for the formation of planetesimals.

Acknowledgements. Authors acknowledge the support of the EU INTAS grant 97-2149.

\section{References}

Bingham, R., \& Tsytovich, V. N. 2001, IEEE Trans. Plasma Sci., 29, 158

Blum, J., et al. 2000, Phys. Rev. Lett., 85, 2426

Boufendi, L., \& Bouchoule, A. 1994, Plasma Sources Sci. Technol., 3, 262

Chevalier, R. A. 1992, Nature, 355, 691

Garscadden, A., Ganguly, B. N., Healand, P. D., \& Williams, J. 1994, Plasma Sources Sci. Technol., 3, 239

Kempf, S., Pfalzner, S., \& Henning, T. 1999, Icarces, 141, 388 Khodataev, Ya. K., Morfill, G. E., \& Tsytovich, V. N. 2001, J. Plasma Phys., in press

Meaking, P. 1991, Rev. Geophys., 29, 317

Morfill, G. E. 2000, Private communication

Spitzer, L. Jr. 1978, Physical Processes in the Interstellar Medium (John Wiley and Sons, New York)

Thomas, H. M., \& Morfill, G. E. 1996, Nature, 379, 806

Tsytovich, V. N., Khodataev, Y., \& Bingham, R. 1996a, Comments on Plasma Physics and Controlled Fusion, 17, 249

Tsytovich, V. N., Khodataev, Y., Bingham, R., \& Tarakanov, V. 1996b, Advances in Dusty Plasma, ed. P. K. Shukla, D. A. Mendis, \& T. Desai, World Scientific, Singapore, 212 Tsytovich, V. N. 1997, Physics Uspekhy, 40, 53

Watson, W. D., \& Salpeter, E. E. 1972, ApJ, 174, 321

Weidenschilling, S. J., \& Cuzzi, J. N. 1993, Protostars and Planets III, ed. E. Levy, \& J. I. Lunine (Univ. Arizona Press, Tucson 1993), 1031

Winters, J. M., Keady, J. J., Gauger, A., \& Sada, P. V. 2000, A\&A, 359, 651 